\title{
INDICADORES FISICOQUÍMICOS Y BIOLÓGICOS DEL PROCESO DE EUTROFIZACIÓN DEL LAGO TITIKAKA (BOLIVIA)
}

\section{PHYSICOCHEMICAL AND BIOLOGICAL INDICATORS OF THE EUTROPHICATION PROCESS AT TITIKAKA LAKE (BOLIVIA)}

\author{
Francisco Fontúrbel Rada ${ }^{1}$
}

\begin{abstract}
Resumen
Se evaluó el grado de avance del proceso de eutrofización del lago Titikaka por medio de la evaluación de las macrófitas (pleuston y limnófitas), de la diversidad de fitoplancton, de parámetros fisicoquímicos $\left(\mathrm{pH}\right.$, turbidez, $\mathrm{DBO}_{5}$, nitrógeno total $\mathrm{y}$ fósforo soluble) $\mathrm{y}$ microbiológicos (coliformes fecales termotolerantes) en cuatro localidades del lago. Los resultados mostraron un avance diferencial en el desarrollo del proceso de eutrofización en cada sitio: se clasificó un sitio como aguas de calidad mínima, un sitio como calidad baja y dos como calidad media. La conjunción de turbidez, $\mathrm{DBO}_{5}$, nitrógeno y fósforo se manifiesta como un buen indicador del grado de avance del proceso de eutrofización, mientras que la evaluación de macrófitas y fitoplancton proporcionan datos valiosos sobre la pérdida de biodiversidad.
\end{abstract}

Palabras clave: eutrofización, eutrofización localizada, lagos, indicadores, macrófitas, fitoplancton, nutrientes, diversidad.

\begin{abstract}
The degree of the advances in the Titikaka Lake eutrophication process was investigated by macrophytes (pleuston and limnophytes) assessment, phytoplankton diversity, physicochemical parameters $\left(\mathrm{pH}\right.$, turbidity, $\mathrm{BOD}_{5}$, total nitrogen and soluble phosphorus) and microbiological (thermotolerant fecal coliforms) parameters at four places of lake. Results showed differential advances in the development of the eutrophication process: one site was classified as minimum water quality, another as low quality water and two as average water quality. Turbidity, $\mathrm{BOD}_{5}$, nitrogen and phosphorus considered jointly are good indicators of the degree of advance of the eutrophication process, whereas macrophyte and phytoplankton assessment provide valuable information on biodiversity loss.
\end{abstract}

Keywords: eutrophication, localized eutrophication, lakes, indicators, macrophytes, phytoplankton, nutrients, diversity.

\section{Introducción}

La eutrofización o hipertrofia es un proceso mayormente de origen antrópico (Smith \& Smith, 2001), que en las últimas décadas ha avanzado considerablemente por el crecimiento de los centros urbanos y el consiguiente aumento en la producción de residuos sólidos y líquidos (Dolbeth et al., 2003; Western 2001), los cuales aumentan la concentración de ciertos nutrientes en cuerpos de agua lénticos (aguas en reposo), ocasionando una degradación del ambiente que, muchas veces, es irreversible (Carpenter \& Cottingham, 1997; Myrbo \& Ito, 2003).

El lago Titikaka no es ajeno a esta problemática, puesto que considerando el acelerado crecimiento demográfico de varias poblaciones ribereñas y el aumento del turismo no planificado en la región, se ha incrementado significativamente la cantidad de residuos sólidos que se descargan a las aguas del Titikaka y lo contaminan, acorde con estudios de Fontúrbel et al. (2003) y Fontúrbel (2004b).
Un estudio de Norticote (1991) dio aviso sobre la proliferación de macrófitas acuáticas flotantes (principalmente Lemna gibba y Azolla sp.) en la parte peruana del Lago, y en Bolivia también se ha reportado la proliferación de estas plantas en los últimos años, pero han sido escasos los estudios que han tratado el tema de la eutrofización, y más aún, de la eutrofización localizada (Fontúrbel, 2004b). Pocos son los estudios que han tratado y documentado el tema para Bolivia, y si bien los procesos eutróficos se dan localizados en sitios puntuales (Fontúrbel, 2003; Fontúrbel, 2004b; Fontúrbel et al., 2003), es apremiante la necesidad de realizar investigaciones sobre el estado y desarrollo de los mismos, especialmente en lugares muy contaminados con procesos eutróficos avanzados como es el caso de Bahía Cohana (Fontúrbel, 2004b).

El objetivo del presente artículo fue estudiar comparativamente el estado de los procesos eutróficos localizados de cuatro localidades de la parte boliviana del Lago Titikaka por medio del análisis de 
indicadores químicos y biológicos (Fontúrbel 2003, 2004b; Schroll, 2002), que permitieron evaluar el estado actual de cada localidad, y clasificarlas en función al avance del proceso eutrófico y de la calidad de aguas (según los Límites Máximos Permisibles de la legislación boliviana, Ley 1333 de Medio Ambiente).

\section{Materiales y métodos}

\section{Área de estudio}

Se muestrearon cuatro localidades de la parte boliviana del Lago Titikaka, cuyas ubicaciones geográficas se detallan en la Tabla 1 y en la Figura 1.

Tabla 1: Ubicación geográfica de los sitios de estudio.

\begin{tabular}{ccc}
\hline Localidad & Coordenadas geográficas & Altitud [m] \\
\hline Cohana & $68^{\circ} 39^{\prime} 36,01^{\prime \prime} \mathrm{W}, 16^{\circ} 22^{\prime} 15,52^{\prime \prime} \mathrm{S}$ & 3843 \\
Copacabana & $69^{\circ} 05^{\prime} 29,11^{\prime \prime} \mathrm{W}, 16^{\circ} 09^{\prime} 50,45^{\prime \prime} \mathrm{S}$ & 3855 \\
Tiquina & $68^{\circ} 50^{\prime} 03,98^{\prime \prime} \mathrm{W}, 16^{\circ} 13^{\prime} 21,90^{\prime \prime} \mathrm{S}$ & 3845 \\
Alaya & $68^{\circ} 48^{\prime} 52,68^{\prime \prime} \mathrm{W}, 15^{\circ} 56^{\prime} 13,48^{\prime \prime} \mathrm{S}$ & 3825 \\
\hline
\end{tabular}

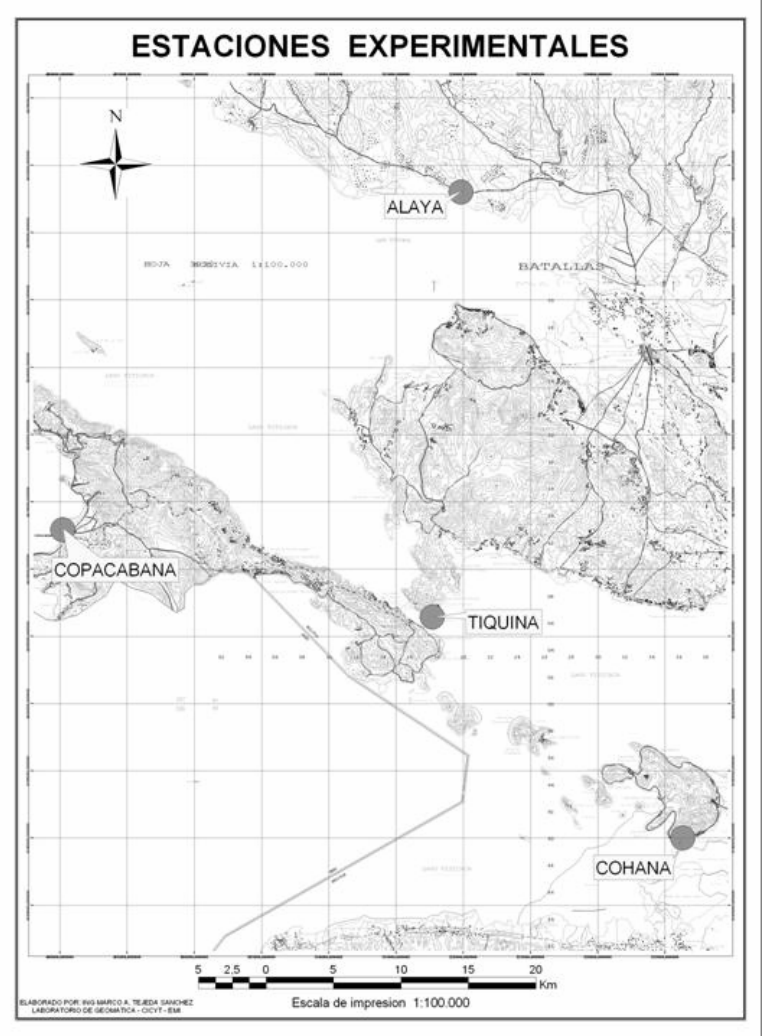

Figura 1. Mapa de ubicación de las áreas de estudio (elaborado por Marco A. Tejada, Laboratorio de Geomática, Escuela Militar de Ingenieria).

\section{Diseño de muestreo}

En cada localidad, se seleccionaron tres áreas de muestreo, cada área de muestreo fue de $100 \mathrm{~m}^{2}$, definidos por un rectángulo de $20 \mathrm{~m}$ a lo largo de la orilla y $5 \mathrm{~m}$ (en perpendicular) hacia adentro del lago, con excepción de Tiquina donde las facilidades del criadero de truchas de la Cooperación Japonesa permitieron trabajar en un área de $140 \mathrm{~m}^{2}$. Para los análisis físico-químicos y biológicos se tomaron aleatoriamente muestras por triplicado de cada área de muestreo. Para la toma de datos se utilizaron formularios de campo previamente diseñados.

Análisis de las macrófitas y del fitoplancton

Se tomaron muestras de las macrófitas flotantes (pleuston) para su posterior identificación en el Herbario Nacional de Bolivia y se estimó la cobertura relativa en superficie en cada área de muestreo. También se tomaron muestras de las macrófitas sumergidas (limnófitas) y se estimó la cobertura relativa de sustrato, así como el índice de diversidad de Shannon-Wiener (Smith \& Smith, 2001). Para el análisis de fitoplancton se tomaron muestras de agua de cada área de muestreo, mismas que fueron fijadas de inmediato mediante solución de lugol y alcohol etílico, para posteriormente ser filtradas $y$ concentradas en laboratorio con una malla de $25 \mathrm{~m}$, para luego realizar el análisis cuali y cuantitativo con ayuda de un microscopio compuesto, en el Laboratorio de Limnología del Instituto de Ecología de la Universidad Mayor de San Andrés. Se estimó la abundancia relativa porcentual y el índice de diversidad de Shannon-Wiener (Smith \& Smith, 2001).

Análisis fisicoquímicos y microbiológicos de aguas

El muestreo de agua para medir el pH se realizó en campo mediante botellas estándar, tomando muestras por triplicado en cada área de muestreo. La lectura de pH del agua se realizó en el Laboratorio de Calidad Ambiental (LCA) mediante el método EPA 150.1. Se tomaron muestras en cada una de las áreas de estudio para determinar turbidez, concentraciones de nitrógeno total (método EPA 351.1), fósforo soluble (método EPA 365.2) y $\mathrm{DBO}_{5}$ (método EPA 405.1). El análisis microbiológico consistió en determinar la cantidad de coliformes termotolerantes (método SM 9221-E). Los valores obtenidos para cada caso, fueron comparados con los Límites Máximos Permisibles fijados por el Reglamento de Contaminación Hídrica de la Ley de Medio Ambiente de Bolivia (Anexo A del Reglamento en Materia de Contaminación Hídrica de la Ley 1333 de Medio Ambiente).

Análisis estadísticos

Se realizaron análisis de varianza (ANOVA) para cada una de las variables mediante el software ESBStats 1.2.3.241 versión estándar, se elaboró una matriz de correlación de Pearson para todos los componentes químicos y biológicos mediante Excel 2003, y se llevó a cabo un análisis multivariable de 
análisis normalizado de componentes principales (ACPn) para elaborar un diagrama de ordenación con las variables como vectores dentro del círculo unitario, por medio de ADE-4 para Windows (Thioluse et al., 1997).

\section{Resultados}

Los resultados del análisis de macrófitas se muestran en la Tabla 2. Los resultados del análisis de fitoplancton se muestran en la Tabla 3.

Tabla 2: Especies de macrófitas (pleuston y limnófitas) registradas en cada uno de los sitios de estudio. Se muestra el índice de diversidad de Shannon-Weiner $\left(\mathrm{H}^{\prime}\right)$ calculado para cada caso.

\begin{tabular}{|c|c|c|c|c|}
\hline \multicolumn{5}{|c|}{ Pleuston } \\
\hline \multirow{2}{*}{ Especie } & \multicolumn{4}{|c|}{ Cobertura relativa [\%] } \\
\hline & Cohana & Copacabana & Tiquina & Alaya \\
\hline Lemna gibba & $20,00 \%$ & $0,00 \%$ & $20,00 \%$ & $0,00 \%$ \\
\hline Nasturtium officinale & $20,00 \%$ & $0,00 \%$ & $0,00 \%$ & $0,00 \%$ \\
\hline H' calculado & 0,694 & indet & 0,000 & indet \\
\hline \multicolumn{5}{|c|}{ Limnófitas } \\
\hline \multirow{2}{*}{ Especie } & \multicolumn{4}{|c|}{ Cobertura relativa [\%] } \\
\hline & Cohana & Copacabana & Tiquina & Alaya \\
\hline Chara fragilis & $0,00 \%$ & $0,00 \%$ & $15,00 \%$ & $0,00 \%$ \\
\hline Chorella sp. & $0,00 \%$ & $0,00 \%$ & $50,00 \%$ & $0,00 \%$ \\
\hline Cladophora sp. & $0,00 \%$ & $45,00 \%$ & $0,00 \%$ & $50,00 \%$ \\
\hline Elodea potamogeton & $0,00 \%$ & $0,00 \%$ & $0,00 \%$ & $2,00 \%$ \\
\hline $\begin{array}{l}\text { Myriophyllum } \\
\text { quitense }\end{array}$ & $30,00 \%$ & $5,00 \%$ & $10,00 \%$ & $40,00 \%$ \\
\hline $\begin{array}{l}\text { Potamogeton } \\
\text { pectinatus }\end{array}$ & $0,00 \%$ & $1,00 \%$ & $0,00 \%$ & $0,00 \%$ \\
\hline $\begin{array}{l}\text { Schoenoplectus } \\
\text { californicus ssp. } \\
\text { Totora }\end{array}$ & $0,00 \%$ & $0,00 \%$ & $35,00 \%$ & $0,00 \%$ \\
\hline $\mathrm{H}^{\prime}$ calculado & 0,000 & 0,420 & 1,335 & 0,774 \\
\hline
\end{tabular}

En la Tabla 4 se muestran los resultados de los análisis físico-químicos realizados (se muestran valores promedio solamente), y también se muestran los resultados obtenidos para el análisis de coliformes termotolerantes. En la Tabla 5 se resumen los resultados del ANOVA. En la Tabla 6 se muestra la matriz de correlación de Pearson de todas las variables.

En la Fig. 2 se presenta el diagrama de ordenación con las variables como vectores dentro del círculo unitario.
Tabla 3. Géneros de fitoplancton registrados en cada uno de los sitios de estudio. Se muestra el índice de diversidad de Shannon-Weiner (H') calculado para cada caso.

\begin{tabular}{|c|c|c|c|c|}
\hline \multirow{5}{*}{ Género } & \multicolumn{4}{|c|}{ Abundancia relativa [\%] } \\
\cline { 2 - 5 } & Cohana & Copacabana & Tiquina & Alaya \\
\hline Anabaena & $0,00 \%$ & $3,28 \%$ & $5,21 \%$ & $0,00 \%$ \\
\hline Asterionella & $0,00 \%$ & $1,64 \%$ & $0,00 \%$ & $4,17 \%$ \\
\hline Ceratium & $0,00 \%$ & $0,00 \%$ & $6,25 \%$ & $0,00 \%$ \\
\hline Closterium & $39,58 \%$ & $4,92 \%$ & $11,46 \%$ & $4,17 \%$ \\
\hline Cosmarium & $0,00 \%$ & $0,00 \%$ & $3,13 \%$ & $0,00 \%$ \\
\hline Diatoma & $0,00 \%$ & $0,00 \%$ & $11,46 \%$ & $0,00 \%$ \\
\hline Diatomella & $0,00 \%$ & $0,00 \%$ & $2,08 \%$ & $0,00 \%$ \\
\hline Fragilaria & $0,00 \%$ & $0,00 \%$ & $1,04 \%$ & $0,00 \%$ \\
\hline Gomphosphaeria & $4,17 \%$ & $0,00 \%$ & $0,00 \%$ & $0,00 \%$ \\
\hline Microcystis & $2,08 \%$ & $0,00 \%$ & $15,63 \%$ & $12,50 \%$ \\
\hline Mougeotia & $25,00 \%$ & $6,56 \%$ & $13,54 \%$ & $45,83 \%$ \\
\hline Navicula & $6,25 \%$ & $0,00 \%$ & $3,13 \%$ & $4,17 \%$ \\
\hline Nodularia & $0,00 \%$ & $9,84 \%$ & $4,17 \%$ & $0,00 \%$ \\
\hline Oscillatoria & $6,25 \%$ & $47,54 \%$ & $12,50 \%$ & $16,67 \%$ \\
\hline Peridinium & $0,00 \%$ & $0,00 \%$ & $2,08 \%$ & $0,00 \%$ \\
\hline Phacus & $2,08 \%$ & $0,00 \%$ & $0,00 \%$ & $0,00 \%$ \\
\hline Pinnularia & $6,25 \%$ & $1,64 \%$ & $0,00 \%$ & $0,00 \%$ \\
\hline Sphaerophea & $0,00 \%$ & $3,28 \%$ & $0,00 \%$ & $0,00 \%$ \\
\hline Spirogyra & $0,00 \%$ & $4,92 \%$ & $0,00 \%$ & $4,17 \%$ \\
\hline Synedra & $0,00 \%$ & $0,00 \%$ & $3,13 \%$ & $0,00 \%$ \\
\hline Tribonema & $0,00 \%$ & $0,00 \%$ & $1,04 \%$ & $0,00 \%$ \\
\hline Ulothrix & $0,00 \%$ & $4,92 \%$ & $0,00 \%$ & $0,00 \%$ \\
\hline Zygnema & $8,33 \%$ & $11,48 \%$ & $4,17 \%$ & $8,33 \%$ \\
\hline H' calculado & $\mathbf{1 , 5 5 2}$ & $\mathbf{1 , 8 2 7}$ & $\mathbf{2 , 4 5 9}$ & $\mathbf{1 , 5 2 5}$ \\
\hline
\end{tabular}

\section{Discusión}

Los resultados obtenidos para las cuatro localidades de estudio muestran diferentes grados de eutrofización debidos -al menos en parte- a las actividades productivas del lugar y cómo se llevan a cabo; En Bahía Cohana se realiza la cría intensiva de ganado, en Tiquina se tiene el criadero de truchas más grande de la parte boliviana del lago, en Copacabana existe intensa actividad turística todo el año y en Alaya existen cultivos hasta el borde de la orilla. Resulta evidente al comparar los datos obtenidos, que los problemas de contaminación y de eutrofización más severos se encuentran en la Bahía de Cohana, en las otras tres estaciones experimentales los problemas son menores, pero no por ello de menor importancia. 
Tabla 4. Valores medidos para los análisis fisicoquímicos y microbiológicos realizados. Solamente se muestran valores promedio. A la derecha se muestra la relación con los Límites Máximos Permisibles (LMP) de la Ley 1333 de Medio Ambiente, donde NE = parámetro no expresado en la legislación.

\begin{tabular}{|c|c|c|c|c|c|c|c|c|}
\hline \multicolumn{9}{|c|}{ Análisis fisicoquímicos y microbiológicos } \\
\hline \multirow{2}{*}{ Parámetro } & \multicolumn{4}{|c|}{ Valor promedio } & \multicolumn{4}{|c|}{ LMP según Ley 1333} \\
\hline & Cohana & Copacabana & Tiquina & Alaya & Clase A & Clase B & Clase C & Clase D \\
\hline $\mathrm{pH}$ & 7.2 & 9.4 & 8.3 & 7.7 & $6,0-8,5$ & $6,0-9,0$ & $6,0-8,5$ & $6,0-9,0$ \\
\hline $\mathrm{DBO}_{5}[\mathrm{mg} / \mathrm{l}]$ & 159.300 & $<0,200$ & $<0,200$ & $<0,200$ & $<2$ & $<5$ & $<20$ & $<30$ \\
\hline $\begin{array}{c}\text { Fósforo soluble } \\
{[\mathrm{mg} / \mathrm{l}]}\end{array}$ & 1.953 & $<0,400$ & 0.001 & 0.054 & 0.4 & 0.5 & 1.0 & 1.0 \\
\hline $\begin{array}{c}\text { Nitrógeno total } \\
{[\mathrm{mg} / \mathrm{l}]}\end{array}$ & 31.900 & 1.170 & 1.010 & 2.400 & 5 & 12 & 12 & 12 \\
\hline $\begin{array}{c}\text { Turbidez } \\
{[\text { ABS@622nm] }}\end{array}$ & 0.088 & 0.000 & 0.000 & 0.025 & $\mathrm{NE}$ & $\mathrm{NE}$ & $\mathrm{NE}$ & $\mathrm{NE}$ \\
\hline $\begin{array}{c}\text { Coliformes } \\
\text { fecales [NMP / } \\
100 \mathrm{ml}]\end{array}$ & 2733 & 370 & 1534 & 5510 & $<50$ & $<1000$ & $<5000$ & $<50000$ \\
\hline
\end{tabular}

es necesariamente la proliferación superficial de Lemna gibba la única responsable de la reducción de la diversidad de las limnófitas, ya que la degradación de los ambientes y el desarrollo de los procesos eutróficos en toda su magnitud, tienen por consecuencia la reducción de la diversidad y la heterogeneidad de las especies (Dolbeth et al., 2003; Fontúrbel, 2004c; McCook, 1999).

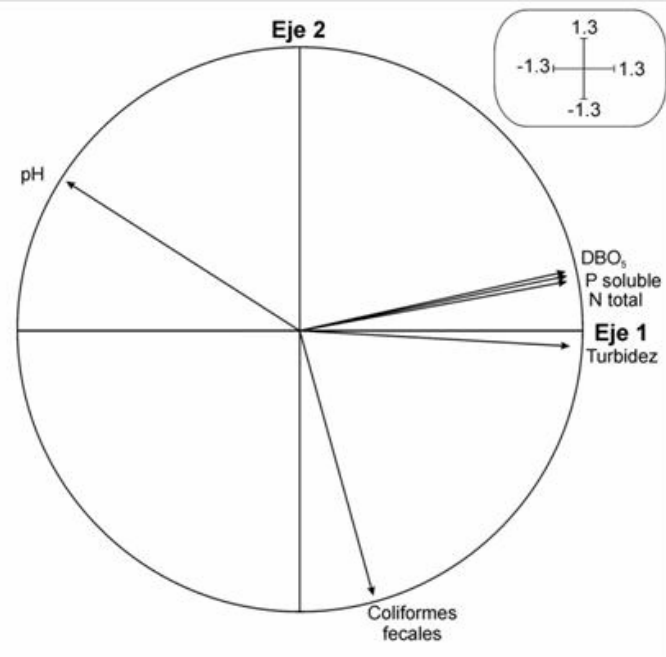

Figura 2: Círculo de correlación de ACPn de los factores fisicoquímicos analizados, obtenido mediante ADE-4 para Windows (redibujado en CorelDRAW 11 por el autor).

La proliferación de macrófitas flotantes (pleuston) -especialmente Lemna gibba- en Cohana y en Tiquina es un claro indicador del avance del proceso eutrófico (Fontúrbel, 2003; Fontúrbel, 2004b; Fontúrbel, 2004c), generalizado en el caso de Cohana y localizado en el caso de Tiquina La aparición de esta flora característica de los procesos eutróficos (García, 2003; Ramsar, 1999; Rodríguez 2002) es en gran parte la responsable de la pérdida de las macrófitas sumergidas (limnófitas), que en el caso de Cohana están prácticamente ausentes, con excepción de unos pocos individuos superficiales de Myriophyllum quitense que también son extraídas para usarse como forraje. Por los resultados mostrados en la Tabla 2, es posible evidenciar la reducción de la diversidad de las macrófitas sumergidas en función a la contaminación y al avance de los procesos eutróficos. El caso de Copacabana (con un índice $H^{\prime}=0,42$ ) sugiere que no
Tabla 5. Resumen de los resultados de ANOVA obtenidos para los datos de análisis fisicoquímicos y microbiológicos.

\begin{tabular}{|c|c|c|c|c|}
\hline \multicolumn{5}{|c}{ Análisis de varianza (ANOVA) } \\
\hline Parámetro & $\begin{array}{c}\text { Intervalo } \\
\text { Confianza }\end{array}$ & $\begin{array}{c}\text { Valor F } \\
\text { calculado }\end{array}$ & $\begin{array}{c}\text { Valor F } \\
\text { tabulado }\end{array}$ & Criterio \\
\hline pH & $95 \%$ & 16,8053 & 4,07 & $\begin{array}{c}\text { Existe } \\
\text { diferencia } \\
\text { significativa }\end{array}$ \\
\hline DBO5 & $95 \%$ & 17,8936 & 4,07 & $\begin{array}{c}\text { Existe } \\
\text { diferencia } \\
\text { significativa }\end{array}$ \\
\hline P soluble & $95 \%$ & 1,3059 & 4,07 & $\begin{array}{c}\text { No existe } \\
\text { diferencia } \\
\text { significativa }\end{array}$ \\
\hline Turbidez & $95 \%$ & 832,6982 & 4,07 & $\begin{array}{c}\text { Existe } \\
\text { diferencia } \\
\text { significativa }\end{array}$ \\
\hline Coliformes \\
fecales
\end{tabular}

Tabla 6. Matriz de correlación de Pearson calculada para los datos de análisis fisicoquímicos y microbiológicos. Se marcan con cursivas los valores de mayor correlación calculada.

\begin{tabular}{|c|c|c|c|c|c|c|}
\hline & $\mathbf{p H}$ & $\mathbf{D B O}_{\mathbf{5}}$ & $\begin{array}{c}\mathbf{N} \\
\text { total }\end{array}$ & $\begin{array}{c}\mathbf{P} \\
\mathbf{s o l u b l} \\
\mathbf{e}\end{array}$ & $\begin{array}{c}\text { Coliforme } \\
\mathbf{s} \text { fecales }\end{array}$ & $\begin{array}{c}\text { Turbide } \\
\mathbf{z}\end{array}$ \\
\hline $\mathbf{p H}$ & 1,000 & & & & & \\
\hline $\mathbf{D B O}_{\mathbf{5}}$ & $-0,669$ & 1,000 & & & & \\
\hline $\mathbf{N}$ total & $-0,689$ & 0,999 & 1,000 & & & \\
\hline P soluble & $-0,690$ & 0,999 & 0,998 & 1,000 & & \\
\hline $\begin{array}{c}\text { Coliforme } \\
\text { s fecales }\end{array}$ & $-0,700$ & 0,059 & 0,098 & 0,070 & 1,000 & \\
\hline Turbidez & $-0,804$ & 0,959 & 0,970 & 0,959 & 0,333 & 1,000 \\
\hline
\end{tabular}

La reducción de la cobertura relativa de Schoenoplectus californicus ssp. totora también es un indicador del avance y la degradación acelerada de los 
ecosistemas acuáticos del lago Titikaka, ya que esta especie es responsable de gran parte de la capacidad de resiliencia del lago, ya que tiene un metabolismo capaz de absorber y filtrar los contaminantes del agua aumentando la capacidad de compensar los daños, aunque no de manera ilimitada (Barrón et al., 2003; Carpenter \& Cottingham, 1997; De Leo \& Levin, 1997; Janssen \& Carpenter, 1999; Rockström et al., 1999). En el caso particular de la Bahía de Cohana es la presencia remanente de esta especie la que todavía mantiene algo de la productividad de la región, ya que $S$. californicus subsp. totora permite reciclar una parte del aporte nutritivo orgánico. La reducción de las limnófitas es un proceso que desestabiliza a todo el ecosistema acuático (McCook, 1999), sin embargo es difícil precisar una causa precisa para este fenómeno, ya que los resultados obtenidos sugieren que la reducción de la cobertura relativa y de la diversidad de las limnófitas estaría en función a la conjunción de varios factores, como es la proliferación de pleuston, el aumento de la turbidez, la inhibición del crecimiento por exceso de nutrientes y materia orgánica, la reducción de la concentración de oxígeno disuelto y la incidencia diferencial de la PAR (radiación fotosintéticamente activa, por sus siglas en inglés), dentro del marco de lo que Western (2001) ha denominado un síndrome de estrés general del ambiente.

En cuanto a los datos de fitoplancton (Tabla 3) es posible apreciar que en los lugares más contaminados existe una menor diversidad de fitoplancton, que de acuerdo con Dolbeth et al. (2003) y Western (2001), sugieren el avance del proceso eutrófico de un nivel inicial a intermedio, o incluso de intermedio a avanzado del proceso eutrófico (Fontúrbel, 2004c). De acuerdo con los indicadores biológicos de Terrel \& Bytnar (1996) existe una marcada predominancia de géneros de fitoplancton característicos de aguas estancadas, de sitios contaminados $y$ de aguas superficiales (en orden de importancia, como por ejemplo Oscillatoria, Mougeotia o Spirogyra).

Las variables fisicoquímicas y microbiológicos se muestran como indicadores bastante certeros para la estimación del avance del proceso eutrófico (Fontúrbel, 2004a ; Valente et al., 1997). De acuerdo a límites máximos permisibles de la legislación boliviana (que establece cuatro categorías de cuerpos de agua: desde $\mathrm{A}$, que corresponde a aguas de máxima calidad y aptas para el consumo humano, hasta $\mathrm{D}$, que corresponde a aguas de mínima calidad y no aptas para el uso humano), se estableció la siguiente clasificación para los sitios de estudio:

- Cohana: Clase D (mínima calidad, no apta para uso humano).

- Alaya: Clase C (baja calidad, potable sólo con tratamiento fisicoquímico completo $\mathrm{y}$ desinfección).
- Copacabana y Tiquina: Clase B (calidad media, potable con tratamiento).

En función a los valores de ANOVA, la matriz de correlación de las Tablas 5 y 6 (respectivamente) y al análisis multivariable que se presenta en la Fig. 2, se puede observar que los parámetros turbidez, $\mathrm{DBO}_{5}$, nitrógeno total y fósforo soluble están relacionados y pueden ser usados en conjunto como indicadores del avance del proceso eutrófico. En función a los resultados mostrados en la Tabla 4, se sugiere el siguiente orden decreciente del grado de eutrofización para las localidades de estudio: Cohana $>$ Alaya $>$ Tiquina $>$ Copacabana. En la Figura 3 se muestra el mapa factorial de las localidades de estudio, obtenido mediante análisis multivariable ACPn.

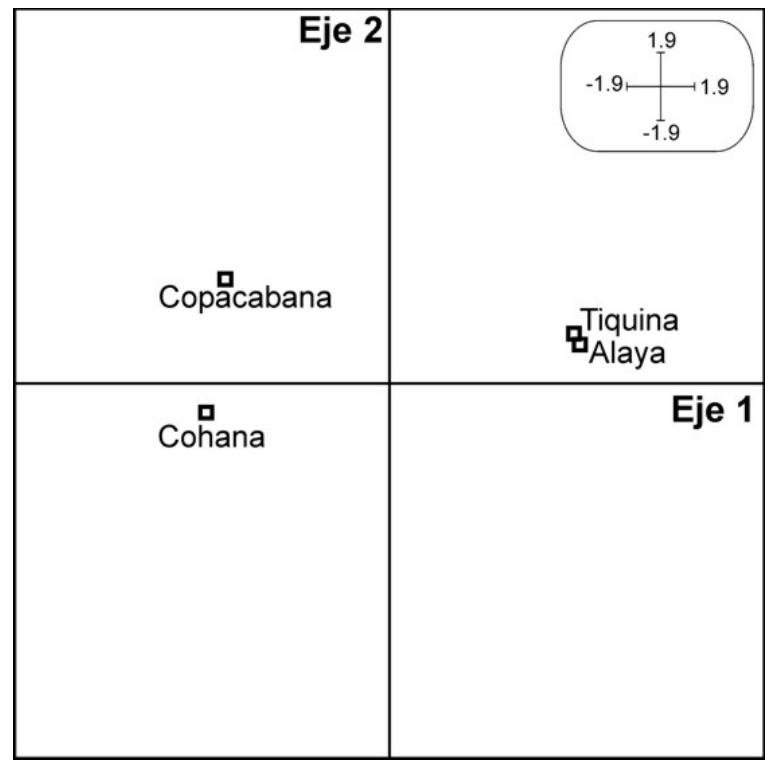

Figura 3. Mapa factorial de localidades de estudio según análisis multivariable ACPn de parámetros fisicoquímicos y microbiológicos, obtenidos mediante ADE-4 para Windows (redibujado en CorelDRAW 11 por el autor).

En el caso de Copacabana es necesario hacer una consideración extra, ya que a pesar de ser un lugar sumamente intervenido y que recibe diariamente la descarga de las aguas servidas de toda la ciudad, es el menos eutrofizado de los cuatro sitios estudiados. Esto puede deberse a que Copacabana es una de las zonas de mayor profundidad de la parte del Lago Mayor, que por ende tiene mayor volumen de agua, permitiendo que la capacidad de resiliencia (por la mayor capacidad de dilución de los contaminantes) sea mucho mayor que en las otras tres localidades, en los que el proceso eutrófico avanza más rápidamente (comparando con datos de Northcote, 1991; Northcote et al., 1989).

La evaluación de coliformes termotolerantes no mostró una correlación muy cercana con las otras variables, pero sirve de indicador de la influencia de la 
descarga de aguas servidas en el lago. Llama la atención el valor tan elevado registrado para Alaya, situación que se puede explicar por la poca profundidad (menos de $5 \mathrm{~m}$ en promedio) de la zona.

\section{Conclusiones}

La aparición y proliferación de macrófitas flotantes (pleuston) muestra estar asociada con la reducción de la diversidad de macrófitas sumergidas (limnófitas) y de fitoplancton. A mayor desarrollo del proceso eutrófico las limnófitas tienden a desaparecer, mientras que la composición de las comunidades de fitoplancton se modifica, quedando uno o dos géneros predominantes (como por ejemplo Oscillatoria y Mougeotia).

Los parámetros $\mathrm{DBO}_{5}$, nitrógeno total, fósforo soluble y turbidez mostraron un elevado grado de correlación y se manifiestan en conjunto como buenos indicadores del grado de eutrofización. Los coliformes termotolerantes se manifiestan como un indicador indirecto relativamente bueno del aporte de aguas servidas al lago. Tanto los parámetros de macrófitas y fitoplancton como los parámetros fisicoquímicos y microbiológicos mostraron ser buenos indicadores del avance del proceso eutrófico localizado en cada uno de los sitios de estudio. Mediante la información obtenida se caracterizó cada localidad según la escala de clases de aguas de la Ley de Medio Ambiente de Bolivia estableciéndose el siguiente orden decreciente del grado de eutrofización: Cohana $>$ Alaya $>$ Tiquina $>$ Copacabana.

Los resultados obtenidos en la presente investigación sugieren un avance de los procesos eutróficos en la parte boliviana del Lago Titikaka, debido principalmente a las actividades antropogénicas y cómo éstas se llevan a cabo, factor que se incrementa a su vez, por el crecimiento demográfico de la región. El creciente aporte de nutrientes a las aguas del lago debería ser controlado para evitar que el proceso de eutrofización siga avanzando, para ello resulta fundamental tomar medidas urgentes de mitigación del problema (especialmente en los sitios más contaminados) e implementar un Plan de Monitoreo Ambiental que sea integral y abarque todo el lago Titikaka.

\section{Agradecimientos}

A Carlos Molina y a la Unidad de Limnología del IE-UMSA por la ayuda brindada durante la fase de análisis de muestras. A Enrique Richard por sus valiosos aportes al manuscrito y por su permanente guía a lo largo del proyecto.

\section{Literatura citada}

Barrón C., Marbà N., Duarte C.M., Pedersen M.F., Lindblat C., Kersting K., Moy F. \& Bokn T. 2003. High organic carbon export precludes eutrophication responses in experimental rocky shore communities. Ecosystems. 6: 144-153.
Carpenter S. \& Cottingham K. 1997. Resilience and Restoration of Lakes. Conservation Ecology. 1, art 2.

De Leo G. \& Levin S. 1997. The multifaceted aspects of ecosystem integrity. Conservation ecology. 1, art. 3.

Dolbeth M., Pardal M.A., Lilleblo A.I., Azeiteiro U. \& Marques J.C. 2003. Short- and long-term effects of eutrophication on the secondary production of an intertidal macrobenthic community. Marine Biology. 101007: 1133-1135.

Fontúrbel F. 2003. Algunos criterios biológicos sobre el proceso de eutrofización a orillas de seis localidades del lago Titikaka. Ecología Aplicada. 2: 75-79.

Fontúrbel F. 2004a. Conservación de ecosistemas: un nuevo paradigma en la conservación de la biodiversidad. Ciencia Abierta Internacional. 23. [online].

Fontúrbel F. 2004b. Modelo operacional ambiental y aspectos sociales relevantes del proceso de eutrofización localizada en cuatro estaciones experimentales del lago Titikaka. Publicaciones Integrales, La Paz.

Fontúrbel F. 2004c. Un ejemplo de la necesidad de la conservación de ecosistemas: la eutrofización localizada en el lago Titikaka (Departamento de La Paz, Bolivia). Revista de Biología.Org. 15, [online].

Fontúrbel F., García G., Brun N., Ferrufino M. \& Rocha R. 2003. Propuesta de producción sostenible de Telmatobius culeus (Anura: Leptodactylidae) en el lago Titikaka, mediante la implementación conjunta de un sistema de control de calidad ambiental. En: Fontúrbel, F. \& G. García (eds.), Propuestas Para Un Desarrollo Sostenible: Lago Titikaka Por Estrategas $K$. Publicaciones Integrales, La Paz. : 19-52.

García P. 2003. Macrófitos acuáticos en los humedales andaluces. Nota de Prensa. Revista Medio Ambiente. Sevilla.

Janssen M. \& Carpenter S. 1999. Managing the Resilience of Lakes: A Multiagent Modeling Approach. Conservation Ecology. 3, art 15.

McCook L.J. 1999. Macroalgae, nutrients and phase shifts on coral reefs: scientific issues and management consequences for the Great Barrier Reef. Coral Reefs. 18: $357-367$.

Myrbo A. \& Ito E. 2003.Eutrophication and remediation in context: High-resolution study of the past 200 years in the sedimentary record of Lake McCarrons (Roseville, Minnesota). USGS-WRRI 104B National Grants Competition and the Center for Agricultural Impacts on Water Quality, Minnesota. : 5.

Northcote T. 1991. Eutrofización y problemas de polución. En: Dejoux, C. \& A. Iltis (eds.), El Lago Titicaca: Síntesis Del Conocimiento Limnológico Actual. Hisbol ORSTOM, La Paz.: 563-572.

Northcote T., Morales P., Levy D. \& Greaven M. 1989.Pollution in lajke Titicaca, Peru: training research and management. Westwater reseach centre, Univ. Brit. Columbia, Vancouver.

Ramsar. 1999. Ramsar COP7 DOC. 24: Especies invasoras y humedales. Ramsar COP, San José.

Rockström J., Gordon L., Folke C., Falkermark M. \& Engwall M. 1999. Linkages among water vapor flows, food production, and terrestrial ecosystem services. Conservation ecology. 3, art. 5.

Rodríguez J.A. 2002.¿Cuál es la flora característica de un proceso eutrófico? Sierra de Baza. Vol. 2003. 
Schroll H. 2002. Indicators of the Long-term Eutrophication of a Danish lake (Karlsø), and Water Pollution Management. The Journal of Transdisciplinary Environmental Studies. 1: 1-10.

Smith R.L. \& Smith T.M. 2001. Ecología. $4^{\circ}$ edición. Addison Wesley, Madrid.

Terrel C. \& Bytnar P. 1996. Water quality indicators guide. Kendall/Hunt publishing company, Dubuque.

Thioluse J., Chessel D., Dolédec S. \& Olivier J.M. 1997. ADE-4: a multivariate analysis and graphical display software. Statistics Comput. 7: 75-83.
Valente J.P., Padilha P. \& Silva A.M. 1997. Contribição da cidade de Botucatu- SP com nutrientes (fósforo e nitrogênio) na eutrofização da represa de Barra Bonita. Eclet. Quim. 22: 1-16.

Western D. 2001. Human-modified ecosystems and future evolution. PNAS. 98: 5458-5465.

\footnotetext{
${ }^{1}$ Institución donde se realizó el trabajo: (La Paz, Bolivia).

Dirección de contacto del autor para correspondencia:

Francisco Fontúrbel R.

Casilla postal \# 180, La Paz, Bolivia

C/ E. Villanueva \# 574, Alto Obrajes Sector A, La Paz, Bolivia.

Tel. +591-2-2731822; +591-72002766

Correo electrónico: fonturbel@gmail.com; fonturbel@yahoo.es
}

Departamento de postgrado, Maestría en Ingeniería de medio ambiente y recursos naturales, Universidad Loyola

Institución actual: Investigador adjunto del Centro de Estudios de Postgrado e Investigación (CEPI) de la Universidad Mayor Real y Pontificia de San Francisco Xavier de Chuquisaca (Sucre, Bolivia). 